\title{
Curcumin Promotes Proliferation of Adult Neural Stem Cells and the Birth of Neurons in Alzheimer's Disease Mice via Notch Signaling Pathway
}

\author{
Jun Li, ${ }^{1}$ Yazhou Han, ${ }^{1}$ Mingduo Li, ${ }^{2}$ and Caixia $\mathrm{Nie}^{3}$
}

\begin{abstract}
The abnormal deposition of amyloid- $\beta$ peptide, a major component of senile plaques, has been reported to be the major cause of neuronal cell death and cognitive impairment in Alzheimer's disease (AD). Adult neurogenesis is related to the amelioration of impaired neurons and cognitive impairment. In the research, we investigated the function of curcumin on endogenous neural stem cells (NSCs) and hippocampal neurogenesis in mice. APP/PS1 transgenic mice as animal models were treated with curcumin, and a significant improvement in learning and memory function was observed. The improvement was associated with a significant increase in the number of new neural stem cells $\left(\mathrm{BrdU}^{+} / \mathrm{Nestin}^{+}\right)$and newborn neurons $\left(\mathrm{NeuN} / \mathrm{kI} 67^{+}\right)$in the hippocampal region and decreased the number of apoptotic neurons ( $\mathrm{TUNEL}^{+}$and Caspase-3/NeuN ${ }^{+}$). These results suggested that curcumin activated NSCs proliferation, improved neurogenesis, and ameliorated cognitive impairment of AD mice. Then, we identified that curcumin upregulated the expression of self-renewal genes, Notch1 and Hes1, and augmentation of CDK4, Cyclin D1, NICD, and Hes1 protein. As Notch activity was blocked by the DAPT, the related proteins were downregulated, and the initiating cell proliferation of curcumin was abolished. These results might suggest that the function of curcumin was dependent on Notch signaling pathway.
\end{abstract}

Keywords: curcumin, Alzheimer's disease, neural stem cells, neurogenesis, apoptosis, cognitive function, neurons, $\gamma$-secretase inhibitor, hippocampus, notch signaling pathway

\section{Introduction}

A LZHEIMER'S DISEASE (AD) is a neurological degenerative disease, and its clinical symptoms are the memory impairment, aphasia, loss of recognition, visual spatial skills impairment, executive dysfunction, and personality and behavioral changes such as dementia. The aggregation of amyloid- $\beta$ peptide $(\mathrm{A} \beta)$ forming amyloid plaques is one of the pathological mechanisms of AD (Joo et al., 2018; Lee et al., 2013; Yang et al., 2018; Yao et al., 2015). The research on hippocampal neurogenesis of central nervous system (CNS) disorders, such as AD, stroke, traumatic brain injury, and so on, found that the endogenous neural stem cells (NSCs) may play regenerative and reparative roles (Sun, 2014; Yan et al., 2018).
However, the transplanted NSCs were limited by low survival rate and neuronal differentiation rate to develop therapeutic strategies (Kong et al., 2015; Zhang et al., 2012; Zhou et al., 2018). Therefore, the study on promoting the NSCs environment and stimulating NSCs neurogenesis is a research hotspot on NSCs dependence therapies of neurological diseases.

Curcumin is a polyphenolic active ingredient extracted from plant turmeric, which has high biological activity. Curcumin has obvious therapeutic effect on AD (Omar et al., 2017). Curcumin can play important roles on reduction of $\mathrm{A} \beta$ oligomer and fibril formation, inhibition of the neurotoxicity of $\mathrm{A} \beta$ in the brain and suppression of inflammation in transgenic mouse brain (Liu et al., 2016; Sun et al., 2017; Sundaram et al., 2017). In addition, many

\footnotetext{
${ }^{1}$ Department of Neurology, Qinyang People's Hospital, Qinyang, China.

${ }^{2}$ Department of Obstetrics and Gynecology, Reproductive Medical Center, Southwest Hospital, Third Military Medical University (Army Medical University), Chongqing, China.

${ }^{3}$ Department of Obstetrics and Gynecology, Reproductive Medical Center, Daping Hospital \& Institute of Surgery Research, Army Medical University (Third Military Medical University), Chongqing, China.
} 
research reported that curcumin can inhibit death of neurons in animal models of neurodegeneration (Feng et al., 2016; Kim et al., 2008; Maiti et al., 2018; Srivastava et al., 2018). Also, some study showed that curcumin strongly activates ERK and p38 kinases to stimulate the proliferation of embryonic cortical neural stem cells through the MAP kinase pathways (Kim et al., 2008).

Notch signaling is a highly conserved signal transduction pathway that plays an important regulatory role in neural stem cell maintenance, proliferation, and differentiation. But it is still unknown whether curcumin could increase endogenous NSCs proliferation of APP/PS1 mice. As the reported function of curcumin, we proposed that curcumin might be a good drug for promoting CNS environment, stimulating endogenous NSC proliferation and neurogenesis, thus efficiently ameliorate cognitive impairment of $\mathrm{AD}$ mice.

\section{Materials and Methods}

\section{Drug preparation}

Curcumin was purchased from the National Institute for the Control of Pharmaceutical and Biological products (Beijing, China), dissolved in polyethylene glycol (PEG $<10 \%$ ) and put in $4^{\circ} \mathrm{C}$ (Tai et al., 2018).

5-Bromo-2-deoxyuridine (BrdU; Sigma Chemical Company, St. Louis, MO) was dissolved in $\mathrm{ddH}_{2} \mathrm{O}$ at $10 \mathrm{mg} / \mathrm{mL}$ and stored at $4^{\circ} \mathrm{C}$. The mice were injected twice per day with $\mathrm{BrdU}(50 \mathrm{mg} / \mathrm{kg}$ body weight, ip.) for 3 days to label cells (Kim et al., 2008; Park et al., 2018).

$\gamma$-Secretase inhibitor $\mathrm{N}$-[N-(3,5-difluorophenacetyl)-Lalanyl]-S-phenylglycine t-butyl ester (DAPT, Sigma Chemical Company) has been identified to interfere with the maintenance and proliferation of NSCs by inhibiting Notch signal pathway (Chojnacki et al., 2003). DAPT was dissolved in DMSO $(<0.1 \%)$, which was used to inject the abdominal of mice as $10 \mathrm{mg} /(\mathrm{kg} \cdot \mathrm{d})$ for seven consecutive days (Borghes et al., 2010).

\section{Animals and animal grouping}

C57BL/6 transgenic APP/PS1 mice, male and female in half, were purchased from the Model Animal Research Center of Nanjing University (Nanjing, China). The APP/PS1 mice contain the overexpressing mutant human Swedish APP $\left(\mathrm{APP}_{\text {swe }}\right)$ and mutant human PS1 (PS1 $\left.\Delta \mathrm{E} 9\right)$, with remarkable elevation of $\beta$-amyloid production associated with certain behavioral abnormalities as AD patients. The transgenic mice were given free access to food and water, maintained at $20^{\circ} \mathrm{C}-25^{\circ} \mathrm{C}$ at room temperature, $40 \%-60 \%$ humidity, and 12 hours daily.

The APP/PS1 mice and wild type were randomly divided into two groups at 9 months, including curcumin-treated ( Tg+Cur) group, Tg group, WT+Cur group, and wild-type (WT) C57BL/6 mice (WT group). The mice in the Tg+Cur group and WT+Cur group were intragastrically adminis- tered with curcumin $(150 \mathrm{mg} / \mathrm{kg})$ dissolved in $10 \%$ PEG daily for 42 days. The mice in the $\mathrm{Tg}$ group were given $10 \%$ PEG only. WT C57BL/6 mice in WT group were given no treatment ( $n=20$ for each group). All experimental protocols were approved by Institutional Animal Care and Use Committee (Jiao et al., 2016; Tai et al., 2018).

\section{Detection of apoptosis in tissues}

Estimation of apoptosis in the tissue was determined using transferase dUTP nick-end labeling (TUNEL) assay and colorimetric TUNEL kit. Mice were anesthetized and perfused with $4 \%$ paraformaldehyde in cold phosphate-buffered saline (PBS). The brains were immediately harvested, snap-frozen in cold isopentane on dry ice, and stored at $-80^{\circ} \mathrm{C}$ until section.

Frozen sections $(5 \mathrm{~mm})$ were prepared using a cryostat microtome. The sections of the brain were fixed with $4 \%$ paraformaldehyde in PBS for 15 minutes. Tissues were permeabilized using $0.2 \%$ Triton $\mathrm{X}-100$. The sections were washed in PBS, and equilibrated for 1 hour using equilibration buffer. Then, they were stained with 4',6-diamidino2-phenylindole (DAPI; Sigma/Aldrich, St. Louis, MO) at $37^{\circ} \mathrm{C}$ for 5 minutes. Finally, fluorescence was recorded on an inverted fluorescence microscope (Nikon). The apoptosis analyses were conducted according to the manufacturer's instruction (San et al., 2018).

\section{Immunohistochemistry}

Brains sections were fixed with $4 \%$ paraformaldehyde in PBS for 30 minutes. After washing twice in PBS, endogenous peroxidase activity was quenched by incubation in $3 \%$ $\mathrm{H}_{2} \mathrm{O}_{2} / 0.1 \%$ Triton X-100 for 30 minutes. Then the sections were incubated with $5 \%$ goat serum to block nonspecific binding for 30 minutes. The sections were incubated at $4^{\circ} \mathrm{C}$ overnight with antibodies against the following proteins: anti-BrdU, mouse monoclonal (1:250); anti-Nestin, rat polyclonal (1:150); anti-Caspase-3, rabbit polyclonal (1:20); and anti-NeuN, mouse polyclonal (1:200) (all from Millipore, Billerica, MA).

After washing with PBS for three times, the sections were incubated with appropriate fluorescein isothiocyanate- or Cy3-conjugated secondary antibodies (1:200; Jackson ImmunoResearch Laboratories, West Grove, PA) for $60 \mathrm{~min}$ utes at room temperature and counterstained with DAPI. For control, the primary antibody was omitted for all markers. Sections were observed by Nikon Eclipse E800 microscope. Fluorescence was recorded on the inverted fluorescence microscope. Fluorescence was analyzed by the ImageJ software.

\section{Real-time polymerase chain reaction}

Real-time polymerase chain reaction (PCR) was performed on an ABI 7500 Real-Time PCR System (Applied Biosystems). The primers are described in Table 1. All PCR amplifications were carried out in $20 \mu \mathrm{L}$ reactions containing

Table 1. Mouse Primers for Real-Time Polymerase Chain Reaction

\begin{tabular}{lll}
\hline Genes & \multicolumn{1}{c}{ Sense $\left(5^{\prime} \rightarrow 3^{\prime}\right)$} & \multicolumn{1}{c}{ Anti-sense $\left(5^{\prime} \rightarrow 3^{\prime}\right)$} \\
\hline Notch 1 & TCG TGT GTC AAG CTG ATG AGG A & GTC CGG CAG CTA CAG GTC ACA A \\
Hes 1 & GCA GAC ATT CTG GAA ATG ACT GTG A & GAG TGC GCA CCT CGG TGT TA \\
$\beta$-Actin & GGC TGT ATT CCC CTC CAT CG & CCA GTT GGT AAC AAT GCC ATG T \\
\hline
\end{tabular}


$2 \mu \mathrm{L}$ of diluted cDNA, $0.4 \mu \mathrm{L}$ of forward and reverse primers, $10 \mu \mathrm{L}$ of TransStart Top Green qPCR SuperMix, $0.4 \mu \mathrm{L}$ of Passive Reference Dye, and $6.8 \mu \mathrm{L}$ of RNase-free water. All reactions and negative controls were performed in triplicate reactions using TransStart Top Green qPCR SuperMix (TransGen Biotech, Beijing, China). The fragments were amplified by the following parameters: initial denaturation at $94^{\circ} \mathrm{C}$ for 5 minutes, 45 cycles of $94^{\circ} \mathrm{C}$ for 30 seconds, $54^{\circ} \mathrm{C}$ for 5 seconds, and $60^{\circ} \mathrm{C}$ for 30 seconds. The results were expressed as $\mathrm{Ct}$ values. Relative changes in gene expression were determined using $\Delta \Delta \mathrm{Ct}$ method, and $\beta$-actin was used as the internal control (Li et al., 2018; Yao et al., 2017).

\section{Western blot analysis}

Cells were collected, washed once in PBS, and lysed with RIPA lysis buffer. After examining the proteins' concentration (Bradford protein assay kit; Bio-Rad), the same amount of proteins were separated by SDS-polyacrylamide gel electrophoresis and transferred onto polyvinylidene difluoride membranes. After blocking in 5\% skim milk, the membrane was incubated with primary antibodies (goat anti- $\beta$-actin 1:1000, Santa Cruz), (rabbit anti-NICD 1:1000, rabbit anti-Hes1 1:1000; Abcam), (rabbit anti-Cyclin D1 1:1000, rabbit-CDK4 1:1000; Cell Signaling Technology [CST]). Secondary horseradish peroxidase-conjugated donkey anti-goat 1:2000, goat anti-rabbit 1:2000 (Santa Cruz) antibody was then performed. The immunoreactivity was developed by ECL western blotting detection reagents (Millipore) (Cao et al., 2018). The results were recorded with a gel image system.

\section{Behavioral tests}

Morris water maze task. Morris water maze was used to detect the capability of learning and memory in mice. All the experiments were performed in a cylindrical pool $1.5 \mathrm{~m}$ in diameter filled with water $\left(24^{\circ} \mathrm{C} \pm 1^{\circ} \mathrm{C}\right)$, and a platform of $10 \mathrm{~cm}$ in diameter was set at a constant position $2 \mathrm{~cm}$ below the water level in the center of one of the four quadrants within the pool. Mice were allowed to swim freely for 1 minute to become habituated to the apparatus.

From the next day, in the hidden platform trials, acquisition trials were carried out two times per day for 5 days. In each trial, mice were placed into the water at a fixed starting position, and the time taken to escape onto the hidden platform and the swimming path length were recorded. Mouse has 90 seconds to find the hidden platform (Yan et al., 2017). If it failed, it was guided to the platform. The mice were allowed to stay on the platform for $20 \mathrm{sec}-$ onds. Performance was tested at 24 hours after the final training day in a probe trial, during which the platform was removed, the mice were placed in the start, and its behavior was observed for 90 seconds (Teng et al., 2014).

Open field test. The open field text box is a white arena with the dimensions of $40 \mathrm{~cm}$ long, $40 \mathrm{~cm}$ wide, and $40 \mathrm{~cm}$ height (Parikh et al., 2018). The mice were gently placed in the central area of the experiment box, the video recording was used to track and trace the animal's movement. The EthoVision XT monitoring analysis software counted the movement distance, movement speed, rest time, and average speed of the mice.

\section{Statistical analyses}

The data are presented as the mean $\pm \mathrm{SD}$. The results were analyzed by one-way analysis of variance (ANOVA) test with Bonferroni test.

\section{Results}

\section{Curcumin alleviated cognitive impairment in APP/PS1 transgenic mice}

To investigate the potential therapeutic function of curcumin on $\mathrm{AD}$, we used Morris Water Maze (MWM) to analyze the promotion of the learning and memory capability of APP/PS1 mice. Spatial learning was expressed as the latency of time spent on finding the escape platform in the water maze. Mean escape latency was significantly longer in Tg group than the WT group (37.57 seconds in the $\mathrm{Tg}$ group vs. 23.75 seconds in the WT group at day 5 , $p<0.01$, Fig. 1A), the curcumin-treated mice exhibited a significantly decrease in the latency ( 37.57 seconds in the $\mathrm{Tg}$ group vs. 27.30 seconds in the $\mathrm{Tg}+\mathrm{Cur}$ group at day 5 , $p<0.01$, Fig. 1A), suggesting that the curcumin-treated $\mathrm{Tg}$ mice showed better cognitive performance.

The probe trial was performed to measure the maintenance of memory function after 24 hours delay. The number of platform crossings was notably reduced in the Tg group compared to the WT group, while the number was significantly improved in the Tg+Cur group (6.71 in the Tg+Cur group vs. 3.14 in the Tg group, $p<0.01$, Fig. 1B). However, no statistically significant difference was found between the WT and the WT+Cur group ( $p>0.05$, Fig. 1A, B).

An open field test was performed to measure the spontaneous motor activities and exploration ability. Total traveling distance, average speed, and duration of time spent in central zone were recorded to analyze the locomotion and exploration (Yao et al., 2015). Results indicated that curcumin-treated APP/PS1 mice showed significantly longer total travel distance and more relative time in the central zone compared to the Tg group ( $p<0.05$, Fig. $1 \mathrm{C}, \mathrm{E})$, while did not affect average speed ( $p>0.05$, Fig. 1D). However, no significant difference was identified between the WT and the WT+Cur group, suggesting that curcumin cannot improve the performance of WT mice ( $p>0.05$, Fig. 1C-E). Therefore, we conclude that curcumin significantly ameliorate the spatial learning and memory capabilities in APP/PS1 transgenic mice, which may be related with promoting proliferation of NSCs and neurogenesis induced by curcumin.

\section{Curcumin reduced neuron apoptosis in APP/PS1 transgenic mice}

To evaluate the effects of curcumin treatment on the neuron apoptosis, brain sections were examined for TUNEL assay (Fig. 2A, C) and Caspase-3/NeuN expression (Fig. 2B, D). The results showed that fewer apoptotic neurons were detected in the Tg group, however, in the WT and the $\mathrm{Tg}+\mathrm{Cur}$ groups, a large number of TUNEL and Caspase $-3^{+} /$ $\mathrm{NeuN}^{+}$cells could be detected in the hippocampus, and the number of apoptotic neurons in the Tg+Cur group was reduced compared with the Tg group (TUNEL ${ }^{+}: 29.87$ in the $\mathrm{Tg}+$ Cur group vs. 40.07 in the Tg group, $p<0.01$; Caspase$3 / \mathrm{NeuN}^{+}: 20.33$ in the $\mathrm{Tg}+\mathrm{Cur}$ group vs. 28.50 in the $\mathrm{Tg}$ 

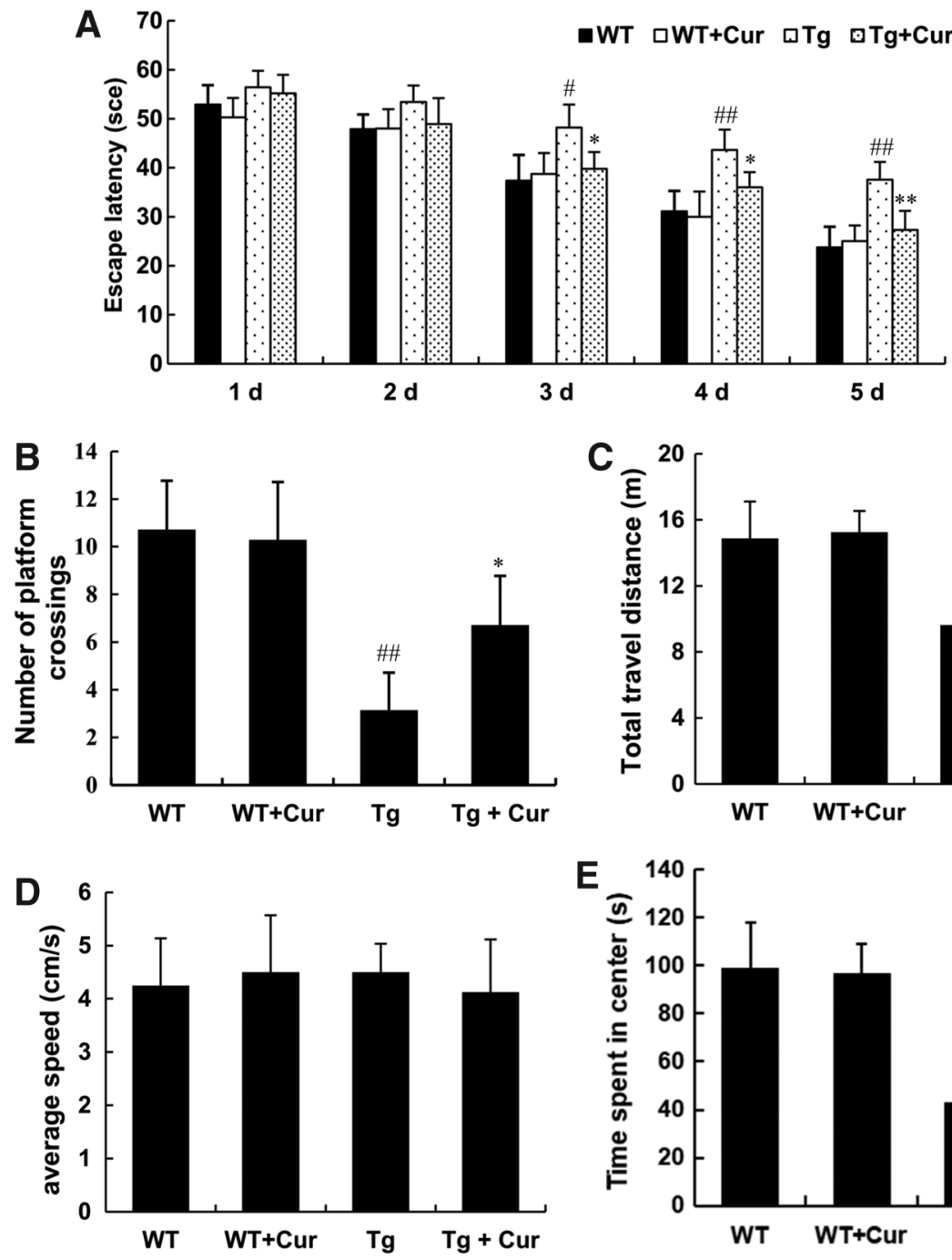
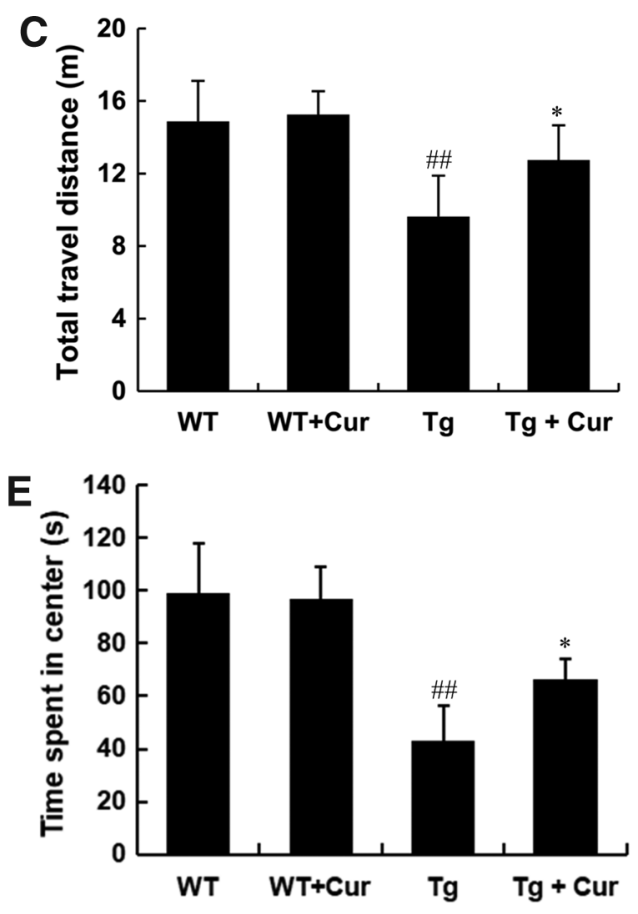

FIG. 1. Curcumin treatment improved cognitive function in APP/PS1 transgenic mice. Morris water maze and open field test was used to evaluate the learning and memory function of the mice. Escape latency in the formal experiments of the water maze task (A). The number of platform crossings (B). Total travel distances (C), average speed (D), and time spent in center (E). ${ }^{\#} p<0.05$ and ${ }^{\# \#} p<0.01$ versus the WT group; $* p<0.05$ and $* * p<0.01$ versus the Tg group. Values are expressed as the mean $\pm \mathrm{SD}$ ( $n=20$ /group). WT, wildtype. group, $p<0.05$, Fig. 2C, D). The results suggested that curcumin could inhibit neuron apoptosis in hippocampus of APP/PS1 mice.

\section{Curcumin promoted neurogenesis in APP/PS1 transgenic mice}

We detected the neurons in hippocampal region after curcumin administration, brain sections were immunostained with antibodies against DCX and Ki67. The number of newborn neurons was found to significantly reduce in the $\mathrm{Tg}$ group $(p<0.01$ vs. the WT group, Fig. 3A, B). On the contrary, treatment with curcumin significantly increased the number of DCX/Ki67-positive cells in APP/PS1 transgenic mice ( $p<0.01$ vs. the Tg group, Fig. 3A, B). Therefore, curcumin is beneficial to hippocampus neurogenesis of APP/PS1 transgenic mice.

\section{Curcumin promoted endogenous NSC proliferation in APP/PS1 transgenic mice}

To identify the proliferation of endogenous NSCs proliferate under curcumin treatment, mice were administered i.p injection of BrdU twice per day $50 \mathrm{mg} / \mathrm{kg}$ for three consecutive days. After curcumin treatment $(150 \mathrm{mg} / \mathrm{kg}$ daily for 6 weeks), the NSCs self-renewal capability in hippocampal region was detected. The proliferation marker BrdU and neural stem cell marker Nestin were detected by immunostaining.

The $\mathrm{BrdU}^{+} / \mathrm{Nestin}^{+}$cells of $\mathrm{Tg}$ group were relatively fewer than that in the WT and the Tg+Cur groups in the dentate gyrus regions of hippocampus. The number of newborn NSCs (BrdU/Nestin ${ }^{+}$) in the Tg group was reduced compared with the WT group (7.27 in the Tg group vs. 20.13 in the WT group, $p<0.01$, Fig. 4A, B), a significant increase was observed in the $\mathrm{Tg}+\mathrm{Cur}$ group (12.87 in the $\mathrm{Tg}+\mathrm{Cur}$ group, vs. 7.27 in the $\mathrm{Tg}$ group, $p<0.01$, Fig. $4 \mathrm{~A}, \mathrm{~B})$. The results suggested that curcumin exposure could enhance the ability of NSCs proliferation in hippocampus of APP/PS1 mice.

\section{Curcumin increases the expression of self-renewal genes in APP/PS1 transgenic mice}

Notch signaling is well known to play a pivotal role in maintaining the characteristics of neural cell. To identify whether curcumin promotes the proliferation of endogenous NSCs through Notch signaling pathway, we used the realtime PCR assay to examine the expression of Notch1 and 
FIG. 2. Curcumin reduced neuron apoptosis in APP/PS1 transgenic mice. Neuron apoptosis as determined by the TUNEL assay and double labeling with antibodies against Caspase-3 and NeuN $(\mathbf{A}, \mathbf{B})$. Scale bar $=50 \mu \mathrm{m}$ in $(\mathbf{A}, \mathbf{B})$. Quantitative analysis of TUNELpositive cells and Caspase-3/NeuNpositive $(\mathbf{C}, \mathbf{D})$. Nuclei were stained with DAPI. ${ }^{\# \#} p<0.01$ versus the WT group; $* p<0.05$ and $* * p<0.01$ versus the $\mathrm{Tg}$ group. Values are expressed as the mean \pm SD $(n=10 /$ group). DAPI, 4',6-diamidino-2phenylindole; TUNEL, transferase dUTP nick-end labeling.
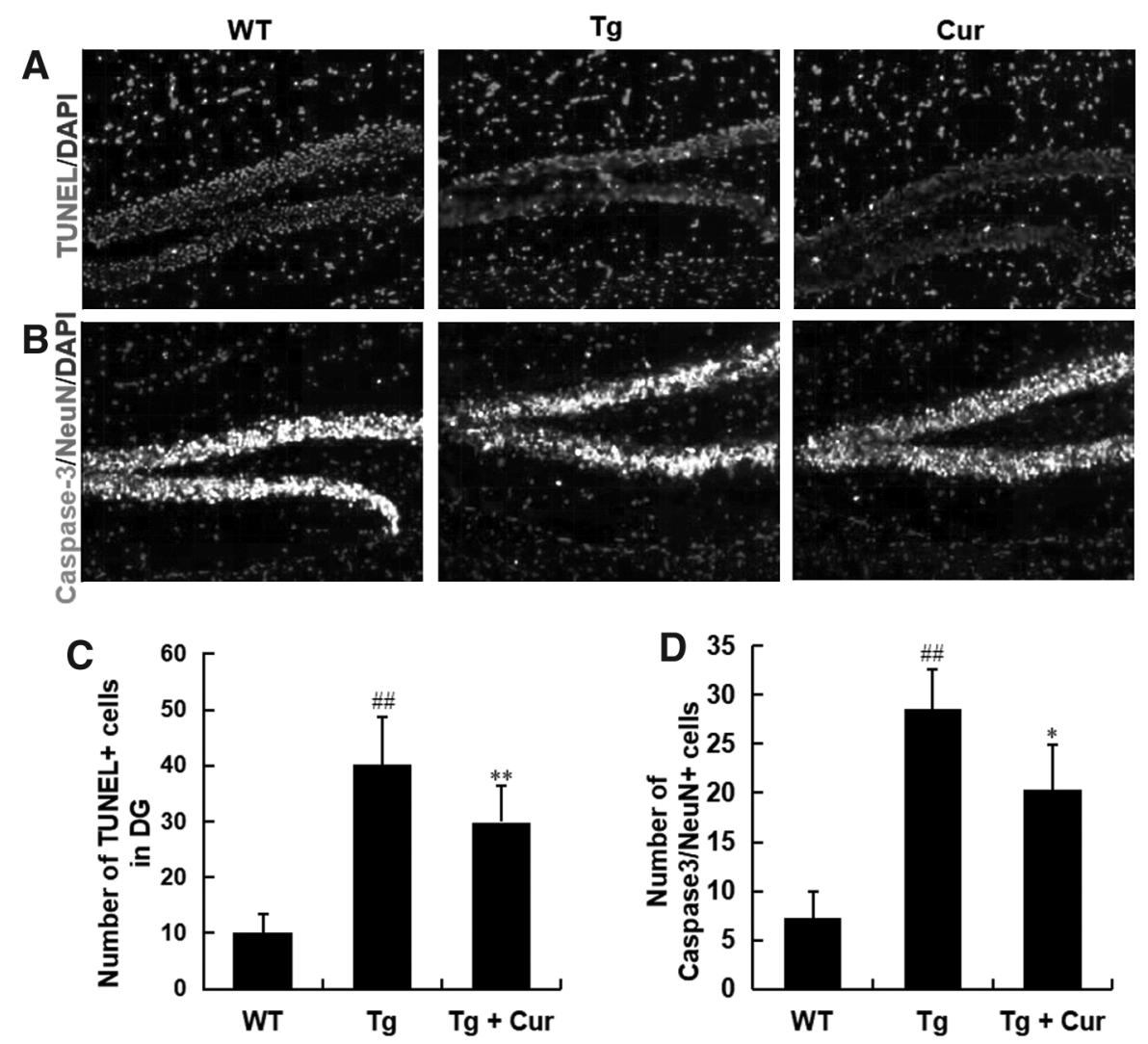

Hes1. The results indicated that the expression levels of Notch1 and Hes1 in the Tg group were lower than that in the WT group (the Tg group vs. the WT group, $p<0.01$, Fig. 5), a significant increasing was observed in the $\mathrm{Tg}+\mathrm{Cur}$ group (the Tg+Cur group vs. the Tg group, $p<0.05$, Fig. 5). The results suggested that curcumin activates Notch pathway and upregulate the expression of self-renewal genes in NSCs.

\section{Curcumin promoted the proliferation of endogenous NSC proliferation through one of Notch signaling way}

To investigate whether the improving of NSCs proliferation of curcumin is through the activation of Notch signaling, the $\gamma$ secretase inhibitor DAPT was performed to block Notch signaling pathway. The proliferation response of curcumin was detected by BrdU/Nestin immunohistochemistry staining. The
A

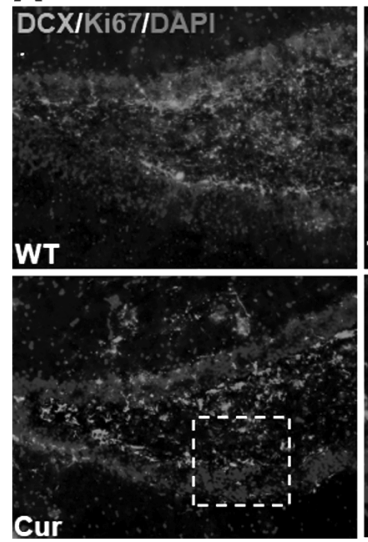

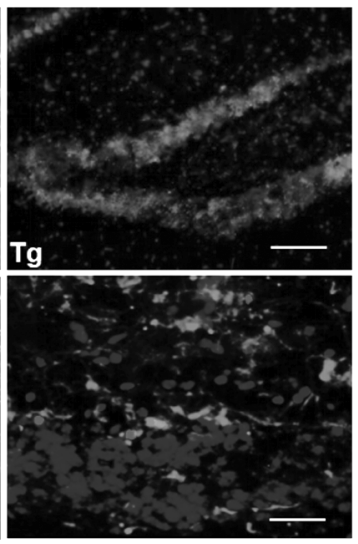

B

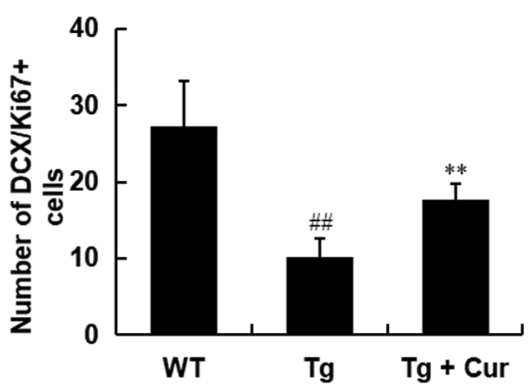

FIG. 3. Curcumin promoted hippocampus neurogenesis in APP/PS1 transgenic mice. Newborn neurons in the hippocampus were detected by immunofluorescent staining with antibody against DCX and Ki67 (A). Quantitative analysis of the number of DCX/Ki67-positive cells (B). Scale bar $=50$ and $25 \mu \mathrm{m}$ in A. Nuclei were stained with DAPI. Double positive cells are shown at higher magnification in the inset. ${ }^{\# \#} p<0.01$ versus the WT group; $* * p<0.01$ versus the Tg group. Values are expressed as the mean $\pm \mathrm{SD}(n=10$ /group). 

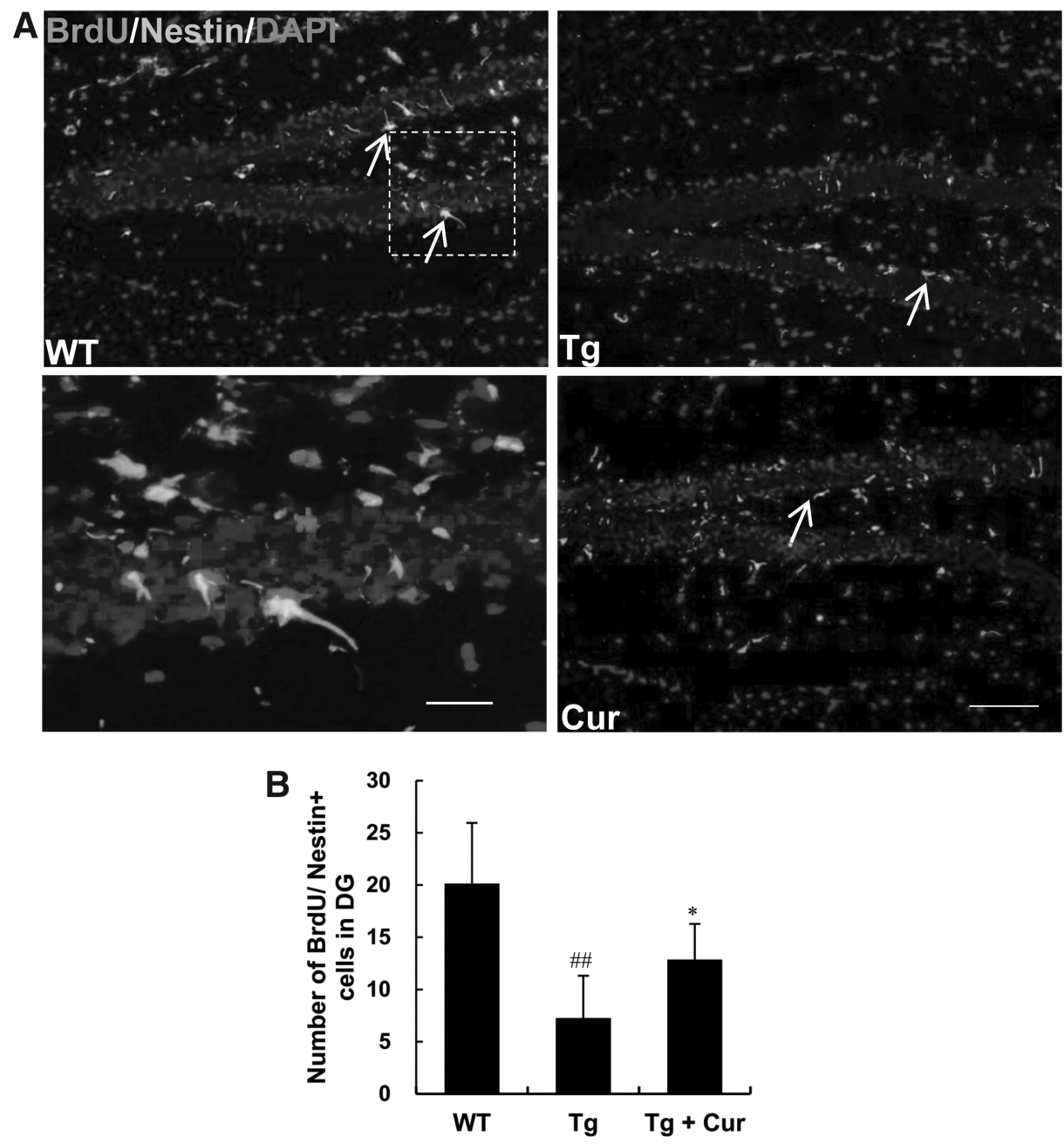

FIG. 4. Curcumin promoted endogenous NSC proliferation in APP/PS1 transgenic mice. Mice received injected BrdU for three consecutive days, Immunostaining with antibodies against BrdU and Nestin to labeled proliferating NSCs in the hippocampus (A). Quantitative analysis of the number of $\mathrm{BrdU}^{+} / \mathrm{Nestin}^{+}$cells (B). Scale $\mathrm{bar}=50$ and $25 \mu \mathrm{m}$ in A. Nuclei were stained with DAPI. The arrows indicate double positive cells. Double positive cells are shown at higher magnification in the inset. $p<0.01$ versus the WT group; $* p<0.05$ versus the $\mathrm{Tg}$ group. Values are expressed as the mean $\pm \mathrm{SD}$ ( $n=10 /$ group). BrdU, 5-bromo-2-deoxyuridine; NSCs, neural stem cells. results showed that curcumin increased the number of $\mathrm{BrdU}^{+} /$ Nestin ${ }^{+}$cells obviously compared with the Tg group. ( $p<0.01$, Fig. 6A, B). Also, blocking Notch signaling pathway by DAPT significantly decreases the number of $\mathrm{BrdU}^{+} / \mathrm{Nestin}^{+}$cells in DAPT groups. No significant difference between the DAPT and the Cur+DAPT group was found ( $p>0.05$, Fig. $6 \mathrm{~A}, \mathrm{~B})$, which

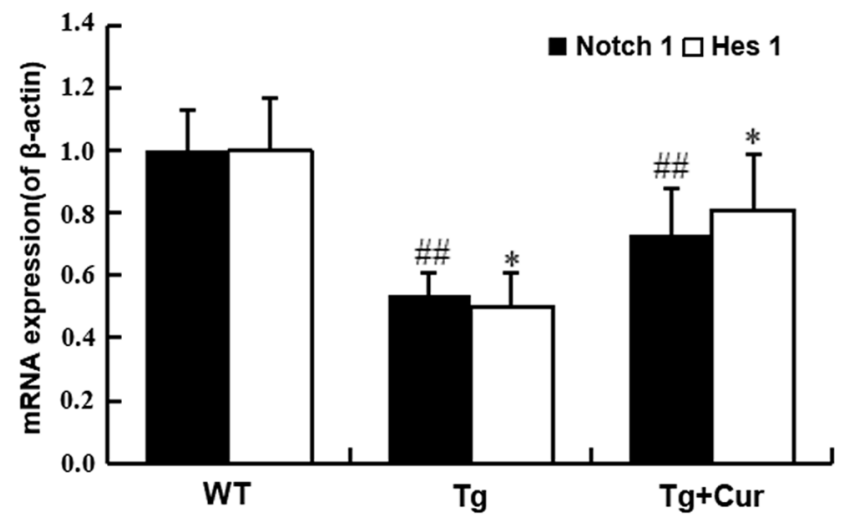

FIG. 5. Curcumin upregulated the expression of selfrenewal genes. The mRNA levels of Notch 1 and Hes 1 were analyzed by real-time PCR. ${ }^{\# \#} p<0.01$ versus the WT group; ${ }^{*} p<0.05$ versus the Tg group. Values are expressed as the mean $\pm \mathrm{SD}$ ( $n=10$ /group). PCR, polymerase chain reaction. indicates that the proliferation-inducing effect of curcumin on endogenous NSCs was abolished by DAPT.

In addition, we used western blotting to detect the expression of CDK4 and Cyclin D1, which showed that curcumin treatment increased CDK4 and Cyclin D1 protein expression compared with the Tg group, however, this upregulation was abolished by Notch pathway inhibitor $(p<0.01$, Fig. 6C-E). To further examine the protein expression of NICD and Hes1 in the curcumin or DAPT group, western blotting showed that curcumin upregulated NICD and Hes1, compared with the Tg group, and this regulation was relieved by DAPT treatment $(p<0.01$, Fig. $6 \mathrm{C}, \mathrm{F})$. These results showed that curcumin promote the proliferation of NSCs through Notch signaling pathway.

\section{Discussion}

In this study, we identified that curcumin can play an important function in promoting the proliferation of NSCs and neurogenesis and the related mechanism in APP/PS1 mouse model. Results revealed that curcumin treatment ameliorated cognitive impairment, reduced neuron apoptosis, promoted endogenous NSCs proliferation, upregulated the Notch1 and Hes1 expression, and augmented protein expression of CDK4, Cyclin D1, NICD, and Hes1. As the activity of Notch was inhibited by $\gamma$-secretase inhibitor 

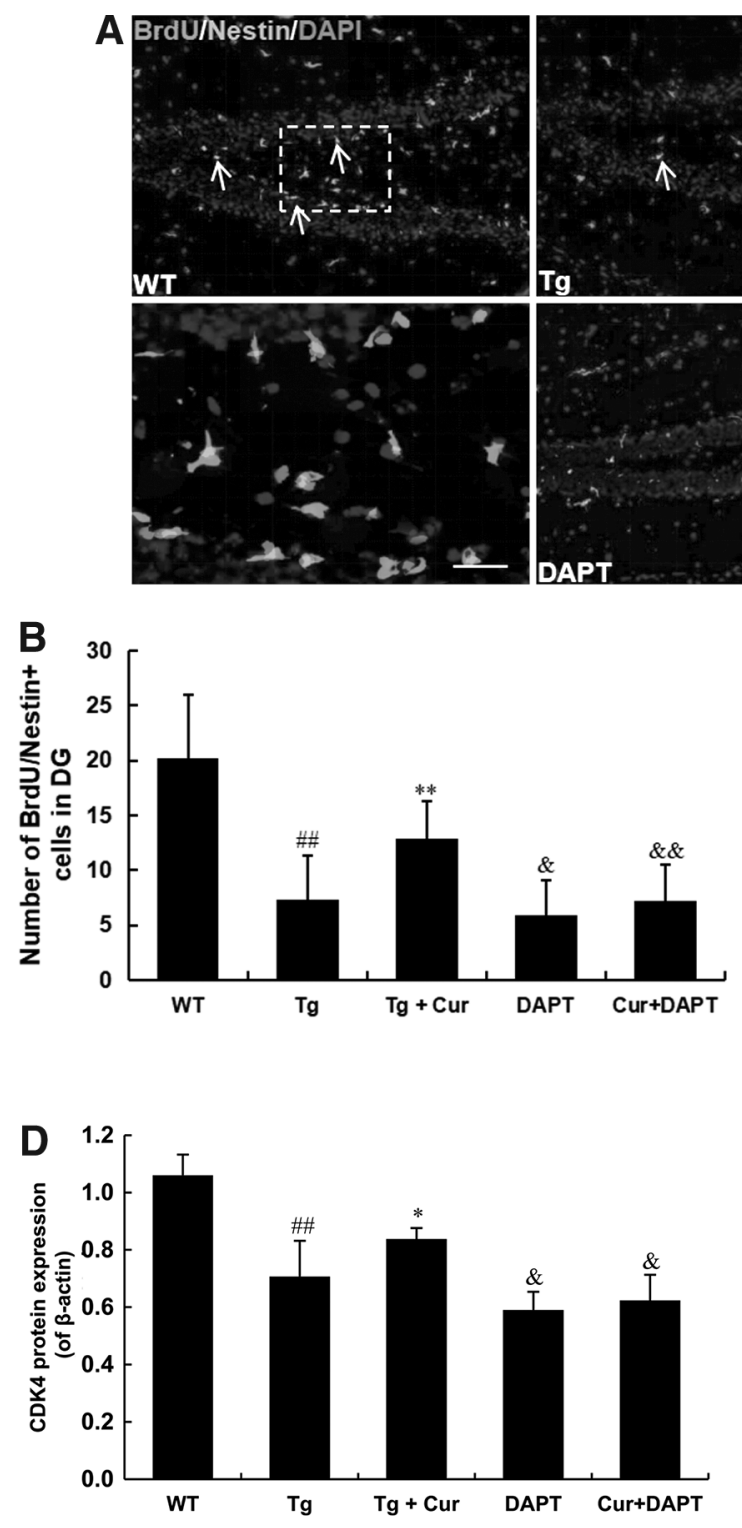

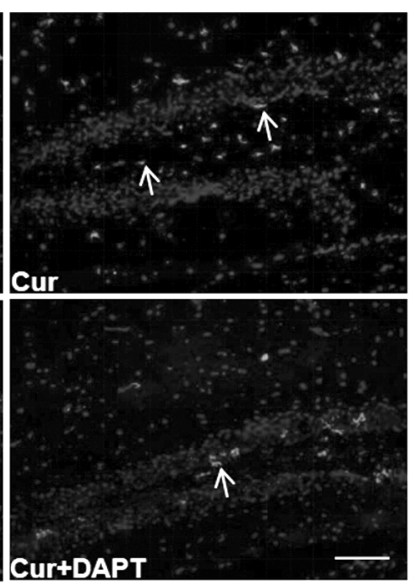

C

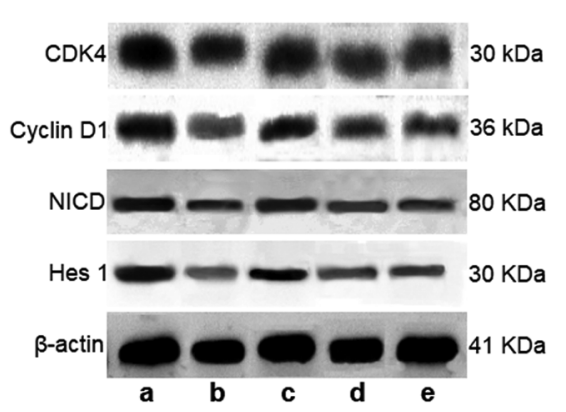

a: WT, b: Tg, c: Tg+Cur, d: DAPT, e: Cur+DAPT

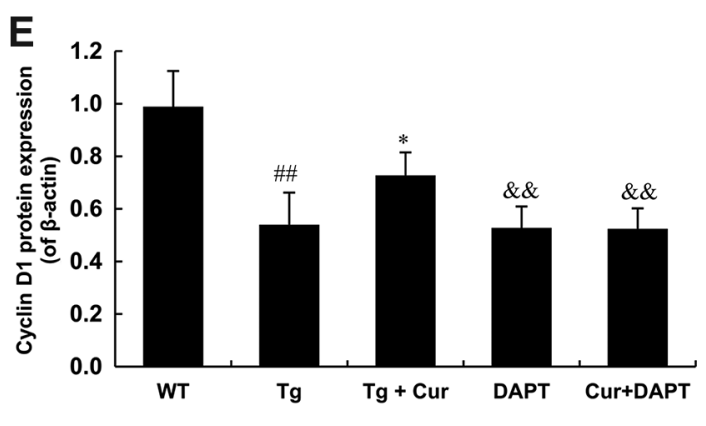

- NICD aHes 1

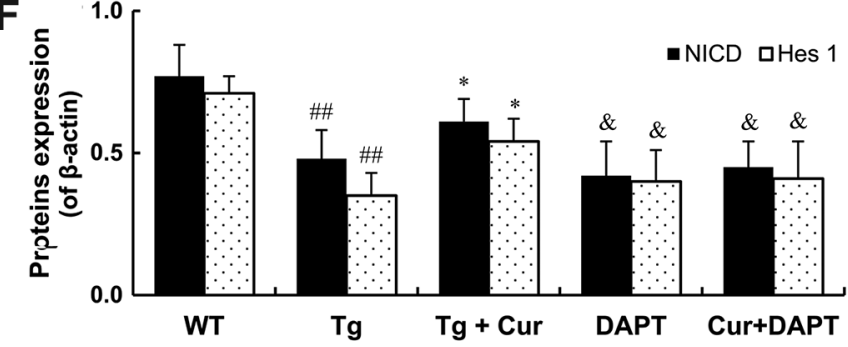

FIG. 6. The proliferation-inducing activity of curcumin is mediated by activation of Notch signaling. Immunostaining with antibody against BrdU and Nestin to labeled proliferating and NSCs in hippocampus in each group (A). Quantitative analysis of the number of $\mathrm{BrdU}^{+} / \mathrm{Nestin}^{+}$cells in each group $(\mathbf{B})$. The protein levels of CDK4, Cyclin D1, NICD, and Hes1 were analyzed by western blot (C). Quantitative analysis of proteins expression (D-F). Scale bar $=50$ and $25 \mu \mathrm{m}$ in A. Nuclei were stained with DAPI. The representative double positive cells are indicated by the arrows and are shown at higher magnification in the inset. ${ }^{\# \#} p<0.01$ versus the WT group; ${ }^{*} p<0.05,{ }^{*} * p<0.0$ versus the Tg group; ${ }^{{ }^{*}} p<0.05,{ }^{\& \&} p<0.0$ versus the Tg+Cur group. Values are expressed as the mean $\pm \mathrm{SD}(n=10$ /group). Tg+Cur, curcumin-treated. 
DAPT, the augmentation of CDK4, Cyclin D1, NICD, and Hes1 protein was ameliorated and the proliferative function of curcumin was blocked. Curcumin was proved to stimulate the proliferation endogenous NSCs proliferation by the activation of Notch pathway.

Alzheimer's is the most common cause of dementia, accounts for $80 \%$ of cases, which leads to the progressive loss of cognitive skills, mental abilities, and learning capability (Anand et al., 2014; Zeng et al., 2018). Altering APP proteolysis is the most obvious feature of Alzheimer's, which produces abnormal $\mathrm{A} \beta$ to induce neurotoxic effects; soluble $\mathrm{A} \beta$ oligomerization is strongly correlated with cognitive decline in AD patients (Kim et al., 2013). This mechanism has been proved to be the major cause of cognitive decline in $\mathrm{AD}$ (Kayed et al., 2003). The neurogenesis in adult may promote the impaired neurons and improve the cognitive impairment in APP/PS1 Tg mice (Deng et al., 2010). Therefore, the therapy of neural stem cells is one of the exciting treatments for neurodegenerative diseases, by replacing the lost neurons (Kim et al., 2006; Zhuang et al., 2012).

Curcumin is a substance in turmeric, which may help to reduce inflammatory, oxidant, and resist infection (Dong et al., 2018; Huang et al., 2018; Ramkumar et al., 2018). Curcumin was reported to have diverse pharmacological functions, such that it could improve cognitive impairments and attenuate cerebral edema (Zhu et al., 2014), and curcumin was reported to enhance cell proliferation in embryonic cortical neural stem cells (Kim et al., 2008; Tiwari et al., 2014). So curcumin may be a promising drug in the therapy of different neuroinflammatory and neurodegenerative disease. In this article, the function of curcumin on proliferation of endogenous NSCs was first investigated, and its impact on hippocampal neurogenesis and hippocampusdependent cognitive functions was further investigated and observed in APP/PS1 Tg mice. It is proved that curcumin could stimulate the proliferation of NSCs in APP/PS1 Tg mice.

To identify that the treatment of curcumin could enhance the proliferation of endogenous NSC in hippocampus, APP/PS1 $\mathrm{Tg}$ mice were injected with $\mathrm{BrdU}$ for three consecutive days. After that, double immunostaining for Nestin and BrdU was used to label the proliferation NSCs. The cells of double immunostaining under curcumin treatment in hippocampus were significantly more than that without treatment in APP/PS1 Tg mice (Fig. 4A, B). In TUNEL and Caspase$3 / \mathrm{NeuN}$ immunofluorescent staining assay, the number of apoptotic neurons in hippocampal region significantly decreased in the $\mathrm{Tg}+\mathrm{Cur}$ group compared with the $\mathrm{Tg}$ group (Fig. 2A-D). On the contrary, curcumin also increased the number of newborn neurons $\left(\mathrm{NeuN} / \mathrm{Ki} 7^{+}\right.$) in the $\mathrm{Tg}+\mathrm{Cur}$ group (Fig. 3A, B). Therefore, curcumin is beneficial to hippocampus neurogenesis of APP/PS1 transgenic mice.

The Notch activity was identified to play an important role in the maintenance of NSCs proliferation and neurogenesis (Imayoshi et al., 2010; Schwanbeck, 2015). For the investigation of the molecular mechanisms of curcumin on the proliferation of NSCs, the relationship between curcumin and Notch signaling pathway was identified. We examined that the mRNA of Notch1 and Hes1 were increased after curcumin treatment under quantitative real-time PCR (qPCR) assay.

These results proved that curcumin initiates Notch signaling pathway by increasing the expression of Notch1 and
Hes1. DAPT, the Notch signaling pathway inhibitor, was used to identify the activation of NSCs proliferation under curcumin treatment, is required with Notch signaling pathway. DAPT significantly inhibited the NSCs proliferation, and the proliferation-inducing effect of curcumin on endogenous NSCs was abolished by DAPT (Fig. 6A, B). The augmented protein expression of Nestin, NICD, and Hes1 induced by curcumin was relieved under DAPT treatment by western blotting assay (Fig. 6C, D).

Cyclin D1 and CDK4, cell cycle proteins, can form a complex known as Cyclin D1/CDK4 to drive cells through the cell cycle G1/S checkpoint to initiate activation (Kim et al., 2014; Liu et al., 2018). We found that curcumin exposure increased the expression of CDK4 and Cyclin D1 protein significantly, and the expression of CDK4 and Cyclin D1 induced by curcumin was relieved by DAPT treatment, which was consistent with the result of BrdU/Nestin, Notch1, and Hes1 protein. These results suggested that the function of curcumin on promoting endogenous NSCs proliferation was dependent on the stimulation of Notch signaling pathway in APP/PS1 transgenic mice.

We also analyzed learning and memory ability of APP/PS1 mice by the Morris water maze and open field test. Our results showed that curcumin could promote the learning and memory functions in the $\mathrm{Tg}+\mathrm{Cur}$ group (Fig. 1), related with curcumin promoting endogenous NSCs and hippocampus neurogenesis, and similar effect was observed in younger APP/PS1 mice (Results not showed).

DAPT is a $\gamma$-secretase inhibitor and inhibits the presenilin $-\gamma$-secretase complex and efficiently inhibits the Notch activity (Wu et al., 2010; Yan et al., 2010). The inhibition of the Notch pathway is primarily dependent on blocking the NICD by $\gamma$-secretase inhibitors, however, it is failed to distinguish individual Notch receptors, inhibit other signaling pathways, thus is associated with numerous complications.

This is not surprising as $\gamma$-secretase is a complex protease compounds with up to 60 potential substrates in addition to Notch (Redmond et al., 2011). Indeed, it is difficult to demonstrate the relationship between NSCs proliferation and Notch activation just using DAPT. Gene modification and siRNAs methods can be applied to selectively block Notch signaling pathways, which confirms whether the proliferation effect of curcumin is depend on the activation of Notch signaling pathway.

Furthermore, many articles reported that some chemicals can replace $\gamma$-secretase directly binding on the nucleotidebinding sites to specifically block cleavage of APP- but not Notch-based substrates, thus selectively regulate the production of $\mathrm{A} \beta$ while sparing Notch (Fraering et al., 2005). We propose that Ost could replace $\gamma$-secretase by directly targeting on a binding position, then modify the receptor of Notch-1 with sparing the APP cleavage. Ost may be a safe drug for the treatment of AD.

To sum up, this research proved that curcumin could stimulate endogenous NSCs proliferation and neurogenesis, which efficiently improved cognitive impairment of APP/PS1 mouse model of Alzheirmer's disease. The function of curcumin on inducing proliferation was further proved to be at least partially dependent on activating Notch signaling pathway. This research suggested that curcumin may be a potential treatment of $\mathrm{AD}$ and neurotrosis. 


\section{Acknowledgment}

This work was supported by Third Military Medical University (Army Medical University) Research Fund. The funders had no role in the study design, data collection or analysis, the decision to publish, or preparation of the article.

\section{Author Disclosure Statement}

The authors declare that no conflicting financial interests exist.

\section{References}

Anand, R., Gill, K.D., and Mahdi, A.A. (2014). Therapeutics of Alzheimer's disease: Past, present and future. Neuropharmacology 76, 27-50.

Borghese, L., Dolezalova, D., Opitz, T., Haupt, S., Leinhaas, A., Steinfarz, B., Koch, P., Edenhofer, F., Hampl, A., and Brüstle, O. (2010). Inhibition of notch signaling in human embryonic stem cell-derived neural stem cells delays G1/S phase transition and accelerates neuronal differentiation in vitro and in vivo. Stem cells 28, 955-964.

Cao, Y., Hölscher, C., Hu, M-M., Wang T., Zhao, F., Bai, Y., Zhang, J., Wu, M-N., and Qi, J-S. (2018). DA5-CH, a novel GLP-1/GIP dual agonist, effectively ameliorates the cognitive impairments and pathology in the APP/PS1 mouse model of Alzheimer's disease. Eur. J. Pharmacol. 827, 215-226.

Chojnacki, A., Shimazaki, T., Gregg, C., Weinmaster, G., and Weiss, S. (2003). Glycoprotein 130 signaling regulates Notch1 expression and activation in the self-renewal of mammalian forebrain neural stem cells. J. Neurosci. 23, 1730-1741.

Deng, W., Aimone, J.B., and Gage, F.H. (2010). New neurons and new memories: How does adult hippocampal neurogenesis affect learning and memory? Nat. Rev. Neurosci. 11, 339-350.

Dong, W., Yang, B., Wang, L., Li, B., Guo, X., Zhang, M., Jiang, Z., Fu, J., Pi, J., Guan, D., and Zhao, R. (2018). Curcumin plays neuroprotective roles against traumatic brain injury partly via Nrf2 signaling. Toxicol. Appl. Pharmacol. 346, 28-36.

Feng, H.L., Dang, H.Z., Fan, H., Chen, X.P., Rao, Y.X., Ren, Y., Yang, J.D., Shi, J., Wang, P.W., and Tian, J.Z. (2016). Curcumin ameliorates insulin signalling pathway in brain of $\mathrm{Alz}-$ heimer's disease transgenic mice. Int. J. Immunopathol. Pharmacol. 29, 734-741.

Fraering, P.C., Ye, W., LaVoie, M.J., Ostaszewski, B.L., Selkoe, D.J., and Wolfe, M.S. (2005). Gamma-Secretase substrate selectivity can be modulated directly via interaction with a nucleotide-binding site. J. Biol. Chem. 280, 41987-41996.

Huang, T., Zhao, J., Guo, D., Pang, H., Zhao, Y., and Song, J. (2018). Curcumin mitigates axonal injury and neuronal cell apoptosis through the PERK/Nrf2 signaling pathway following diffuse axonal injury. Neuroreport 29, 661-677.

Imayoshi, I., Sakamoto, M., Yamaguchi, M., Mori, K., and Kageyama, R. (2010). Essential roles of Notch signaling in maintenance of neural stem cells in developing and adult brains. J. Neurosci. 30, 3489-3498.

Jiao, Y., Kong, L., Yao, Y., Li, S., Tao, Z., Yan, Y., and Yang, J. (2016). Osthole decreases beta amyloid levels through upregulation of miR-107 in Alzheimer's disease. Neuropharmacology 108, 332-344.

Joo, S.H., Yun, S.H., Kang, D.W., Hahn, C.T., Lim, H.K., and Lee, C.U. (2018). Body mass index in mild cognitive impairment according to age, sex, cognitive intervention, and hypertension and risk of progression to Alzheimer's disease. Front. Psychiatry 9, 142.

Kayed, R., Head, E., Thompson, J.L., McIntire, T.M., Milton, S.C., Cotman, C.W., and Glabe, C.G. (2003). Common structure of soluble amyloid oligomers implies common mechanism of pathogenesis. Science 300, 486-489.

Kim, H.T., Kim, I.S., Lee, I.S., Lee, J.P., Snyder, E.Y. and Park, K.I. (2006). Human neurospheres derived from the fetal central nervous system are regionally and temporally specified but are not committed. Exp. Neurol. 199, 222-235.

Kim, M.S., Kim, K.H., Lee, E.H., Lee, Y.M., Lee, S.H., Kim, H.D., and Kim, Y.Z. (2014). Results of immunohistochemical staining for cell cycle regulators predict the recurrence of atypical meningiomas. J. Neurosurg. 121, 1189-1200.

Kim, S.J., Son, T.G., Park, H.R., Park, M., Kim, M.S., Kim, H.S., Chung, H.Y., Mattson, M.P., and Lee, J. (2008). Curcumin stimulates proliferation of embryonic neural progenitor cells and neurogenesis in the adult hippocampus. J. Biol. Chem. 283, 14497-14505.

Kim, S.U., Lee, H.J., and Kim, Y.B. (2013). Neural stem cellbased treatment for neurodegenerative diseases. Neuropathology 33, 491-504.

Kong, L., Hu, Y., Yao, Y., Jiao, Y., Li, S., and Yang, J. (2015). The coumarin derivative osthole stimulates adult neural stem cells, promotes neurogenesis in the hippocampus, and ameliorates cognitive impairment in APP/PS1 transgenic mice. Biol. Pharm. Bull. 38, 1290-1301.

Lee, I.S., Jung, K., Kim, I.S., and Park, K.I. (2013). Amyloid- $\beta$ oligomers regulate the properties of human neural stem cells through GSK-3 $\beta$ signaling. Exp. Mol. Med. 45, e60.

Li, Y., Guan, S., Liu, C., Chen, X., Zhu, Y., Xie, Y., Wang, J., Ji, X., Li, L., Li, Z., Zhang, Y., Zeng, X., and Li, M. (2018). Neuroprotective effects of Coptis chinensis Franch polysaccharide on amyloid-beta $(\mathrm{A} \beta)$-induced toxicity in a transgenic Caenorhabditis elegans model of Alzheimer's disease (AD). Int. J. Biol. Macromol. 113, 991-995.

Liu, W., Wu, W., Lin, G., Cheng, Y., and Shi, Y. (2018). Physical exercise promotes proliferation and differentiation of endogenous neural stem cells via ERK in rats with cerebral infarction. Mol. Med. Rep. 18, 1455-1464.

Liu, Z.J., Li, Z.H., Liu, L., Tang, W.X., Wang, Y., Dong, M.R., and Xiao, C. (2016). Curcumin attenuates beta-amyloidinduced neuroinflammation via activation of peroxisome proliferator-activated receptor-gamma function in a rat model of Alzheimer's disease. Front. Pharmacol. 7, 261.

Maiti, P., Paladugu, L., and Dunbar, G.L. (2018). Solid lipid curcumin particles provide greater anti-amyloid, antiinflammatory and neuroprotective effects than curcumin in the 5xFAD mouse model of Alzheimer's disease. BMC Neurosci. 19, 7.

Omar, S.H., Scott, C.J., Hamlin, A.S., and Obied, H.K. (2017). The protective role of plant biophenols in mechanisms of Alzheimer's disease. J. Nutr. Biochem. 47, 1-20.

Parikh, A., Kathawala, K., Li, J., Chen, C., Shan, Z., Cao, X., Zhou, X.F., and Garg, S. (2018). Curcumin-loaded selfnanomicellizing solid dispersion system: Part II: In vivo safety and efficacy assessment against behavior deficit in Alzheimer disease. Drug. Deliv. Transl. Res. 8, 1406-1420.

Park, J.H., Shin, B.N., Ahn, J.H., Cho, J.H., Lee, T.K., Lee, J.C., Jeon, Y.H., Kang, I.J., Yoo, K.Y., Hwang, I.K., Lee, C.H., Noh, Y.H., Kim, S.S., Won, M.H., and Kim, J.D. (2018). Glehnia littoralis extract promotes neurogenesis in the hippocampal dentate gyrus of the adult mouse through increasing expressions of brain-derived neurotrophic factor and tropomyosinrelated kinase B. Chin. Med. J. (Engl). 131, 689-695.

Ramkumar, M., Rajasankar, S., Gobi, V.V., Janakiraman, U., Manivasagam, T., Thenmozhi, A.J., Essa, M.M., Chidambaram, R., Chidambaram, S.B., and Guillemin, GJ. (2018). De- 
methoxycurcumin, a natural derivative of curcumin abrogates rotenone-induced dopamine depletion and motor deficits by its antioxidative and anti-inflammatory properties in Parkinsonian rats. Pharmacogn. Mag. 14, 9-16.

Redmond, E.M., Guha, S., Walls, D., and Cahill, PA. (2011). Investigational Notch and Hedgehog inhibitors-Therapies for cardiovascular disease. Expert. Opin. Investig. Drugs. 20, 1649-1664.

Sang, Q., Sun, D., Chen, Z., and Zhao, W. (2018). NGF and $\mathrm{PI} 3 \mathrm{~K} /$ Akt signaling participate in the ventral motor neuronal protection of curcumin in sciatic nerve injury rat models. Biomed. Pharmacother. 103, 1146-1153.

Schwanbeck, R. (2015). The role of epigenetic mechanisms in notch signaling during development. J. Cell. Physiol. 230, 969-981.

Srivastava, P., Dhuriya, Y.K., Kumar, V., Srivastava, A., Gupta, R., Shukla, R.K., Yadav, R.S., Dwivedi, H.N., Pant, A.B., and Khanna, V.K. (2018). PI3K/Akt/GSK3 $\beta$ induced CREB activation ameliorates arsenic mediated alterations in NMDA receptors and associated signaling in rat hippocampus: Neuroprotective role of curcumin. Neurotoxicology 67, 190-205.

Sun, D. (2014). The potential of endogenous neurogenesis for brain repair and regeneration following traumatic brain injury. Neural Regen. Res. 9, 688-692.

Sun, J., Zhang, X., Wang, C., Teng, Z., and Li, Y. (2017). Curcumin decreases hyperphosphorylation if Tau by downregulating caveolin-1/GSK-3 $\beta$ in N2a/APP695swe cells and APP/PS1 double transgenic Alzheimer's disease Mice. Am. J. Chin. Med. 45, 1667-1682.

Sundaram, J.R., Poore, C.P., Sulaimee, N.H.B., Pareek, T., Cheong, W.F., Wenk, M.R., Pant, H.C., Frautschy, S.A., Low, C.M., and Kesavapany, S. (2017). Curcumin ameliorates neuroinflammation, neurodegeneration, and memory deficits in p25 transgenic mouse model that bears hallmarks of Alzheimer's disease. J. Alzheimers Dis. 60, 1429-1442.

Tai, Y.L., Lin, Y.Y., Wang, K.C., Chang, C.L., Chen, R.Y., Wu, C.C., and Cheng, T.H. (2018). Curcuminoid submicron particle ameliorates cognitive deficits and decreases amyloid pathology in Alzheimer's disease mouse model. Oncotarget 9, 10681-10697.

Teng, Y., Zhang, M.Q., Wang, W., Liu, L.T., Zhou, L.M., Miao, S.K., and Wan, L.H. (2014). Compound danshen tablet ameliorated $\mathrm{A} \beta_{25-35}$-induced spatial memory impairment in mice via rescuing imbalance between cytokines and neurotrophins. BMC Complement. Altern. Med. 14, 23.

Tiwari, S.K., Agarwal, S., Seth, B., Yadav, A., Nair, S., Bhatnagar, P., Karmakar, M., Kumari, M., Chauhan, L.K., Patel, D.K., Srivastava, V., Singh, D., Gupta, S.K., Tripathi, A., Chaturvedi, R.K., and Gupta, K.C. (2014). Curcumin-loaded nanoparticles potently induce adult neurogenesis and reverse cognitive deficits in Alzheimer's disease model via canonical Wnt/ $\beta$-catenin pathway. ACS Nano. 8, 76-103.

Wu, Y., Cain-Hom, C., Choy, L., Hagenbeek, T.J., de Leon, G.P., Chen, Y., Finkle, D., Venook, R., Wu, X., Ridgway, J., Schahin-Reed, D., Dow, GJ., Shelton, A., Stawicki, S., Watts, R.J., Zhang, J., Choy, R., Howard, P., Kadyk, L., Yan, M., Zha, J., Callahan, C.A., Hymowitz, S.G., and Siebel, C.W. (2010). Therapeutic antibody targeting of individual Notch receptors. Nature 464, 1052-1057.

Yan, M., Callahan, C.A., Beyer, J.C., Allamneni, K.P., Zhang, G., Ridgway, J.B., Niessen, K., and Plowman, G.D. (2010). Chronic DLL4 blockade induces vascular neoplasms. Nature 463, E6-E7.

Yan, Y., Kong, L., Xia, Y., Liang, W., Wang, L., Song, J., Yao, Y., Lin, Y., and Yang, J. (2018). Osthole promotes endogenous neural stem cell proliferation and improved neurological function through Notch signaling pathway in mice acute mechanical brain injury. Brain Behav. Immun. 67, 118-129. Yan, Y.H., Li, S.H., Li, H.Y., Lin, Y., and Yang, J.X. (2017). Osthole protects bone marrow-derived neural stem cells from oxidative damage through PI3K/Akt-1 pathway. Neurochem. Res. 42, 398-405.

Yang, B., Liu, B., Liu, Y., Han, H., and Kuang, H. (2018). Cognitive enhancement of volatile oil from the stems of Schisandra chinensis Baill. in Alzheimer's disease rats. Can. J. Physiol. Pharmacol. 1, 1-6.

Yao, P.L., Zhuo, S., Mei, H., Chen, X.F., Li, N., Zhu, T.F., Chen, S.T., Wang, J.M., Hou, R.X., and Le, Y.Y. (2017). Androgen alleviates neurotoxicity of $\beta$-amyloid peptide $(\mathrm{A} \beta)$ by promoting microglial clearance of $\mathrm{A} \beta$ and inhibiting microglial inflammatory response to A $\beta$. CNS Neurosci. Ther. 23, 855-865.

Yao, X.Q., Jiao, S.S., Saadipour, K., Zeng, F., Wang, Q.H., Zhu, C., Shen, L.L., Zeng, G.H., Liang, C.R., Wang, J., Liu, Y.H., Hou, H.Y., Xu, X., Su, Y.P., Fan, X.T., Xiao, H.L., Lue, L.F., Zeng, Y.Q., Giunta, B., Zhong, J.H., Walker, D.G., Zhou, H.D., Tan, J., Zhou, X.F., and Wang, Y.J. (2015). p75NTR ectodomain is a physiological neuroprotective molecule against amyloid-beta toxicity in the brain of Alzheimer's disease. Mol. Psychiatry 20, 1301-1310.

Yao, Y., Gao, Z., Liang, W., Kong, L., Jiao, Y., Li, S., Tao, Z., Yan, Y., and Yang, J. (2015). Osthole promotes neuronal differentiation and inhibits apoptosis via Wnt/beta-catenin signaling in an Alzheimer's disease model. Toxicol. Appl. Pharmacol. 289, 474-481.

Zeng, Y.Q., Cui, Y.B., Gu, J.H., Liang, C., and Zhou, X.F. (2018). Scutellarin mitigates $A \beta$-induced neurotoxicity and improves behavior impairments in AD mice. Molecules 23. pii: E869. DOI: 10.3390/molecules23040869.

Zhang, N., Kang, T., Xia, Y., Wen, Q., Zhang, X., Li, H., Hu, Y., Hao, H., Zhao, D., Sun, D., Yan, Y., Zhang, G.X., and Yang, J. (2012). Effects of salvianolic acid B on survival, self-renewal and neuronal differentiation of bone marrow derived neural stem cells. Eur. J. Pharmacol. 697, 32-39.

Zhou, C.L., Zhao, L., Shi, H.Y., Liu, J.W., Shi, J.W., Kan, B.H., Li, Z., Yu, J.C., and Han, J.X. (2018). Combined acupuncture and HuangDiSan treatment affects behavior and synaptophysin levels in the hippocampus of senescence-accelerated mouse prone 8 after neural stem cell transplantation. Neural Regen. Res. 13, 541-548.

Zhu, H.T., Bian, C., Yuan, J.C., Chu, W.H., Xiang, X., Chen, F., Wang, C.S., Feng, H., and Lin, J.K. (2014). Curcumin attenuates acute inflammatory injury by inhibiting the TLR4/ MyD88/NF-kappaB signaling pathway in experimental traumatic brain injury. J. Neuroinflammation 11, 59.

Zhuang, P., Zhang, Y., Cui, G., Bian, Y., Zhang, M., Zhang, J., Liu, Y., Yang, X., Isaiah, A.O., Lin, Y., and Jiang, Y. (2012). Direct stimulation of adult neural stem/progenitor cells in vitro and neurogenesis in vivo by salvianolic acid B. PLoS One 7, e35636.

Address correspondence to: Caixia Nie Department of Obstetrics and Gynecology Reproductive Medical Center Daping Hospital \& Institute of Surgery Research Army Medical University (Third Military Medical University) Chongqing 400042 China

E-mail: caixianie1212@163.com 